\title{
Enabling Assistive Technologies to Shape the Future of the Intensive Senior-Centred Care: A Case Study Approach
}

\author{
Adriana ALEXANDRU, Marilena IANCULESCU* \\ National Institute for Research and Development in Informatics, \\ 8-10 Averescu Avenue, Bucharest, 011455, Romania \\ adriana@ici.ro,manina@ici.ro (*Corresponding author)
}

\begin{abstract}
Nowadays, the proportion in the older ages and the average life expectancy is increasing dramatically throughout the world. The health problems associated with wealthy and aged populations has been an issue of great concern to a broad and expanding number of people. Appropriate healthcare ITC-based technologies and services have to cope with this situation. Some specific challenges related to senior-centred care as well as impediments to acceptance of health-related digital approaches by elderly persons are underlined in this paper. The assistive technologies play a major role in increasing, maintaining; or improving the functional capabilities of seniors as well as in creating the opportunities for ageing in place. This paper aims to illustrate how an ongoing eHealth solution can be further upgraded by integrating some of the latest assistive technologies devices. A brief presentation of some representative devices (such as wearable sensors, mHealth apps and inhome monitoring system) highlights their benefits for the senior healthcare delivery. The current phase of the "Conceptual Model for a Platform of Integrated Services based on Cloud for Home Monitoring of Seniors affected by Dementia (MSIMDD)" project is put in. A proposed extended architecture of MSI-MDD platform is provided emphasizing the benefits of the integration of the most appropriate assistive technologies for an improved remote senior-centered healthcare service delivery.
\end{abstract}

Keywords: Senior-centred care, Health-assistive technologies, Wearable technology, mHealth, In-home monitoring.

\section{Introduction}

The global ageing population is increasingly growing coupled with a major increase in life expectancy, due to the improvements in healthcare service delivery, food supply and nutrition, but also due to the innovation and developments in technology and science.

"Compared to 2017, the number of persons aged 60 or above is expected to more than double by 2050 and to more than triple by 2100 . In Europe, $25 \%$ of the population is already aged 60 years or over. That proportion is projected to reach $35 \%$ in 2050. Globally, the number of persons aged 80 or over is projected to triple by 2050." [22]

A growing number of seniors has an increased impact on the whole levels of society, from the individual changing position inside his/her family, to the new national and international strategies, programmes or approaches aiming to best tackle the challenges and demands brought by the ageing population.

Longer life expectancy and incoming number of seniors with associated multimorbidity have dramatically raised the cost associated with the health and social systems, which is more and more often perceived as a burden on social insurance programs, healthcare service delivery or pension systems.
One of the most important issue and concern in this respect is to identify and implement the most appropriate healthcare services and technology which are adapted to the degenerative disorders of the seniors, but also to the demands and needs of so called "tech-savvy" new generations of elderly people that are accustomed and willing to use the digital technology.

A 2017 report from Ericsson ConsumerLab representing the results of two surveys carried out in Germany, Japan, South Korea, the UK and the USA revealed that over 60 percent of surveyed consumers are prepared to include assistive technology as a preventive means for health management. Assistive technology can provide the medical specialists with the opportunities to have access and process the clinically-relevant, real-time health information and data. "As improved healthcare helps people live longer, healthcare systems come under increasing pressure as older patients need more care.

The progressive evolution of age-related disorders have also a negative impact on the seniors' quality of life and independence.

The resulting resource shortage is forcing the industry to become more and more dependent 


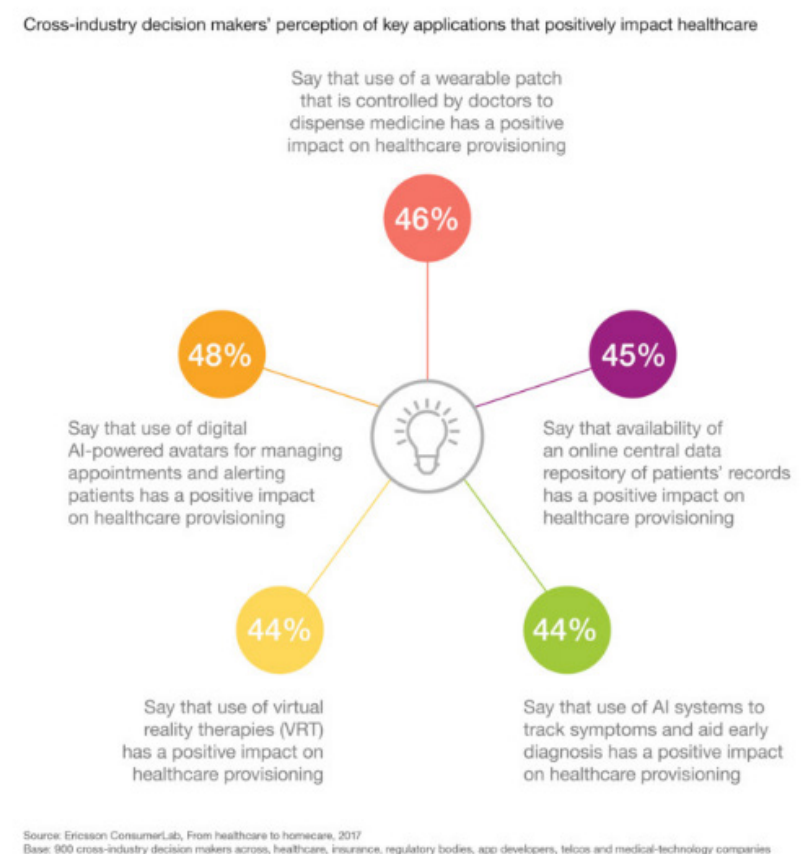

Figure 1. Cross-industry decision makers' perception of key applications that positively impact healthcare [10]

on automation, remote treatment and artificial intelligence (AI)" [10]. See Figure 1.

Chapter 2 introduces the holistic approach of senior-centred care that focuses on enhancing the dimensions of senior's health and well-being. Some specific challenges and the main constraints related to the acceptance and use of consumer-oriented health digital technologies are further detailed.

In Chapter 3, the assistive technologies and healthcare associated applications for seniors' use are presented, with emphasis on wearable sensors, mHealth apps and in-home monitoring systems.

Chapter 4 presents a case study and provides details regarding the modules included in the "Conceptual Model for a Platform of Integrated Services based on Cloud for Home Monitoring of Seniors affected by Dementia (MSI-MDD)" project.

Chapter 5 discusses the proposed future functions to be included in the previously mentioned project and the new associated proposed architecture. These are in line with the assistive technologies and applications presented in Chapter 3 .

\section{Issues related to senior-centred care}

Senior people are more likely to be subject of agerelated health impairments, which can fluctuate in many directions ranging from cognitive decline or mobility dysfunctions to chronic diseases. Therefore the need of expenditure, facilities and access to appropriate and personalised healthcare services is significant and is gaining importance in the context of world ageing population.

Senior-centred care focuses on the elderly' $\mathrm{s}$ health management, taking into consideration any support or health service delivery that might be necessary for ensuring and facilitating a proper life and health condition of the senior.

Specific challenges have to be taken into consideration in order to provide seniorcentred care that can facilitate them a dignified, independent and active life:

- Providing an increased, adapted and affordable assistance with their activities of daily living.

- Training a greater number of better specialised health carer able to respond to the particular necessities of the elderly.

- Endorsing the shift from reactive care to preventive care.

- Sustaining the implementation of new and emerging supporting technologies that aim 
to create a familiar and safe environment and to avoid as much as possible the institutionalisation of the seniors.

- Working out a holistic approach of the degenerative diseases, their associated comorbidities and of the diversity of digital technology in order to provide an improved health management.

- Anticipating the demands of the new generations of seniors which are supposed to be more accustomed and receptive to healthcare supported by digital technology because they already have the appropriate level of health and information technology literacy.

Digital healthcare technology proved undoubtedly to have the potential to support the provision of an integrated senior-centred care across the whole healthcare and social systems and to tackle the challenges of ageing.

Main constraints for a broader acceptance of health digital technology by the seniors:

- Lack of confidence regarding their own capacity to gain knowledge and skills about how to use it.

- Unconvinced position regarding the opportunities and benefits of the involvement of digital technology in the personal health management.

- Physical age-related disabilities that might hinder a proper use of health digital technology.

- Cognitive disorders that limit the total or partial access to the functions provided by the health digital technology.

- Fear about the unauthorised use of the personal data and information.

- Insufficient age-friendly features of the health digital technology.

- Unaffordable cost of some digital devices.

Taking into consideration the fact that the number of the seniors is increasing, their health condition or needs are continuously changing, that they have becoming more empowered and motivated to remain independent, engaged in activities inside the family or community and more determined to age-in-place instead of living in nursing homes, it is imperative that innovations and new technological solutions to be deployed.

Assistive technology for seniors represents a reliable support designed to replace, supplement or recover the diminished or lost physical or cognitive functions and skills of the elderly, helping them to avoid many degradations related to the quality of life.

\section{Some representative health- assistive technologies focusing on a better senior-centred care}

The role of assistive technologies is to:

- Provide support in gaining enhanced accessibility and improved aids for people with physical or cognitive disorders and disabilities.

- Enable seniors to continue their lives in a secure and familiar habitat.

- Reduce negative feelings like insecurity, vulnerability, loneliness, and depression.

- Enable social activity and even working process [1].

- Prevent injuries or accelerated deterioration of health conditions.

"Assistive technology is an umbrella term referring to specialized technology used by people to adapt how specific tasks are performed. Ambient assisted living (AAL) technology is the result of a progression from individual devices assisting with one task or activity of daily living to ambient systems in which the assistance or support completely encompasses the living area and the person". [6] Assistive technology has evolved with and emerged from information technology, passing from detecting and reporting problems, to preventing them (see Figure 2).

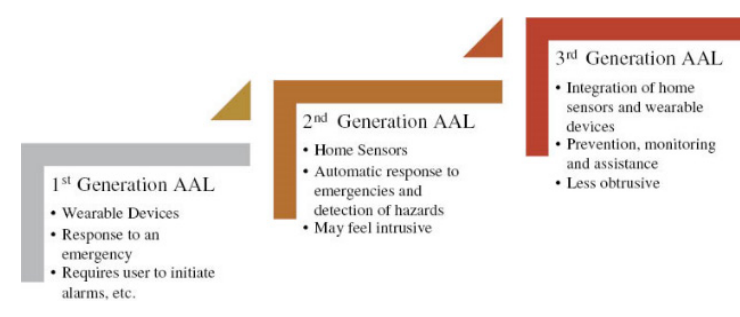

Figure 2. Three Generations of Ambient Assisted Living (AAL) [6] 
Table 1. The Most Representative Assistive Technologies for Aged Care [3]

\begin{tabular}{|l|l|}
\hline \multicolumn{1}{|c|}{ Category } & \multicolumn{1}{c|}{ Most Significant Assistive Technologies } \\
\hline $\begin{array}{l}\text { New medical } \\
\text { devices and sensor } \\
\text { technologies }\end{array}$ & $\begin{array}{l}\text { Non-invasive medical devices. } \\
\text { Wrist watch for personalised monitoring of signs like breathing and pulse, as well as } \\
\text { pathology. } \\
\text { Data visualisation and imaging. }\end{array}$ \\
\hline $\begin{array}{l}\text { Regenerative } \\
\text { Medicine }\end{array}$ & $\begin{array}{l}\text { Nano-based therapies that cross blood-brain barrier. } \\
\text { Stem cells and induced pluripotent stem cells (iPS) technologies. } \\
\text { Biomimetics or the use of smart materials and design inspired by nature. }\end{array}$ \\
\hline $\begin{array}{l}\text { Smart homes and } \\
\text { intelligent materials }\end{array}$ & $\begin{array}{l}\text { Implantable monitoring devices. } \\
\text { Smart fabrics and intelligent materials } \\
\text { Assistive devices designed for low dexterity. } \\
\text { Data monitoring - client controlled. } \\
\text { Smart grids able to withstand power interruptions. } \\
\text { Sources of renewable energy. }\end{array}$ \\
\hline $\begin{array}{l}\text { Integrated health } \\
\text { and social support } \\
\text { services }\end{array}$ & $\begin{array}{l}\text { Integrated Healthcare - streamline care, sharing of documentation. } \\
\text { Smart homes - voice and face activated. } \\
\text { Uptake enablers such as funding, development, design, resources and program } \\
\text { support, and education for carers, individual and professionals. }\end{array}$ \\
\hline $\begin{array}{l}\text { Bio-mechanical } \\
\text { technologies, } \\
\text { robotics and neuro- } \\
\text { prostheses }\end{array}$ & $\begin{array}{l}\text { Neuro-prosthesis - Brain signals have an impact on sensors. } \\
\text { Robots - integrated information systems enabling monitoring, and social } \\
\text { engagement. } \\
\text { Biomechanics - e.g. walking-aid shoes; exoskeletons that help movement. }\end{array}$ \\
\hline
\end{tabular}

The most representative assistive technologies focusing on the seniors are presented in Table 1.

\subsection{Wearable technologies}

Wearable technologies or wearables aim to monitor, prevent or assist the treatment and daily activities of a person; they can be attached in a diverse way to the person or to a currently used item or equipment. Wearables that are focused on seniors have the potential to monitor the vital signs, location or safety of the elderly, to prevent and trigger alerts in case of an emergency situation like a fall or hypertension crisis, to maximize the independence of the seniors and also to support a remote healthcare management.

The actual capabilities of the wearables comprise physiological and biochemical sensing and also motion sensing [7].

In order to decrease the possible restraints of the seniors related to the acceptance of the wearables, many of them can be unobtrusively integrated into "smart" fabrics.

"Currently, smartwatch, smartphone and smart clothing are the mainstream products embedded wearable technologies with care functions. All of them have attractive advantages for delivering health information." [23].
"Smart wearables are becoming increasingly pervasive driven by the continuous miniaturisation of electronics; advances in sensor technology, computing power and connectivity; and, an ever stronger capability to embed intelligence in electronic (and photonic) components and systems, ultimately coupled by a reduction in the price of components." [11]. See Figure 3.

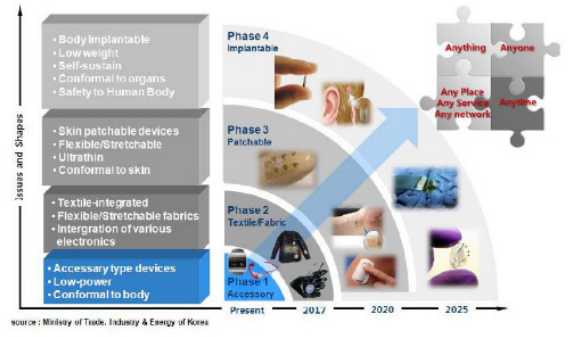

Figure 3. Wearables' evolution [11]

Here are examples of some of the latest wearables appropriate for seniors:

- Thread-based diagnostic platform Considered to be an active substrate for the newest implantable diagnostic devices and smart wearables, the platform is composed of nano-scale sensors, electronics and microfluidics comprised into threads that can be sutured via many layers of tissues (see Figure 4). The health data is gathered wirelessly in real time and it determines a diagnostic like status of healing wound [19]. 
- Smart contact lens - Smart contact lens are designed as a self-powered biosensor for diverse health signs monitoring and wireless biomedical sensing, able to detect in real time the pathogen, bacteria, glucose, and infectious keratitis present in tear fluid [5]. See Figure 5.

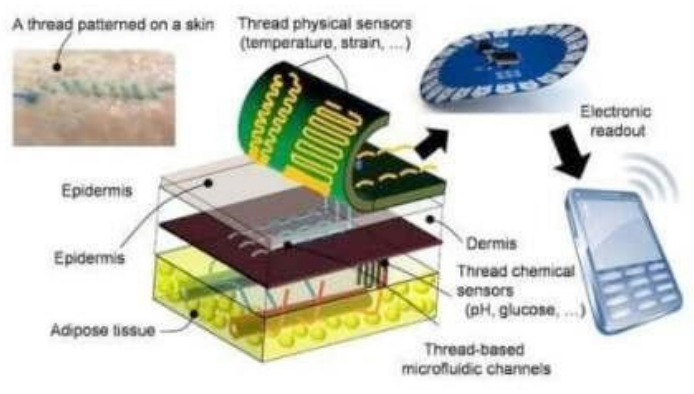

Figure 4. The structure of the thread-based diagnostic platform [19]

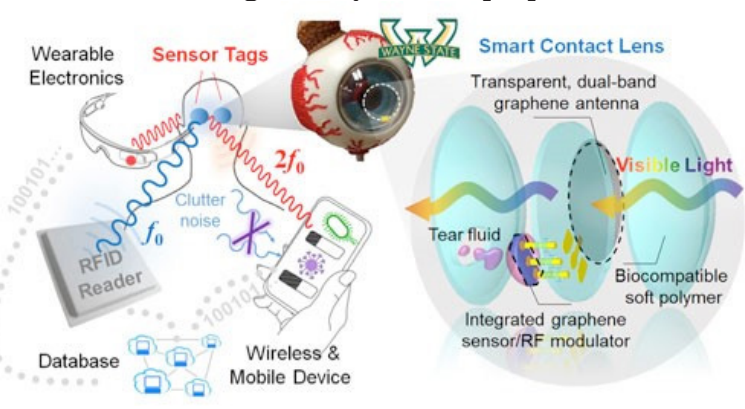

Figure 5. Schematics of smart contact lens [5]

- Human stress monitoring patch - It integrates three sensors that are designed to detect the human physiological signals: skin temperature, skin conductance, and pulse wave in the size of stamp. The skin contact area is minimized through the invention of an integrated multi-layer structure and the associated microfabrication process. The stress is measured by multimodal physiological data analysis [25]. See Figure 6.

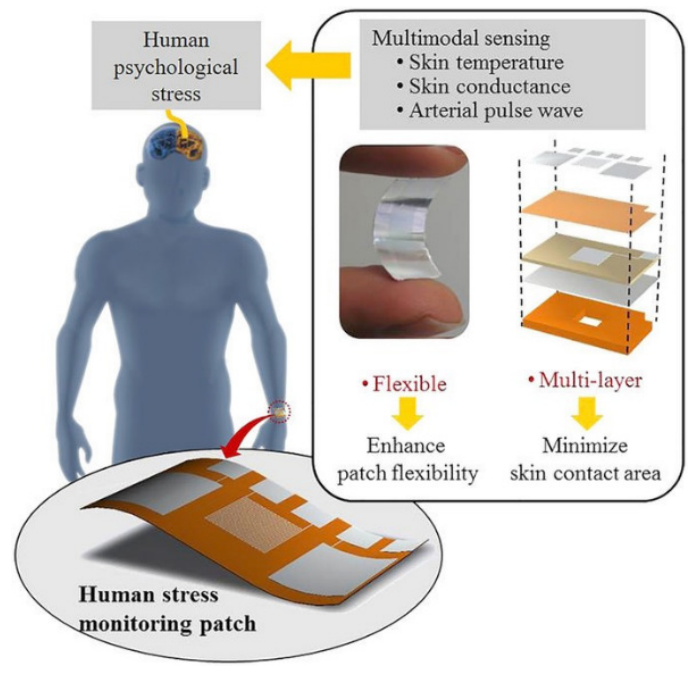

Figure 6. Human stress monitoring patch [25]
- Graphene smart patch for monitoring diabetes - The graphene made patch senses glucose in sweat. A connected portable electrochemical analyser receives data by wires. When blood sugar is too high, it releases the drug metformin through microneedles to lower it [24]. See Figure 7.

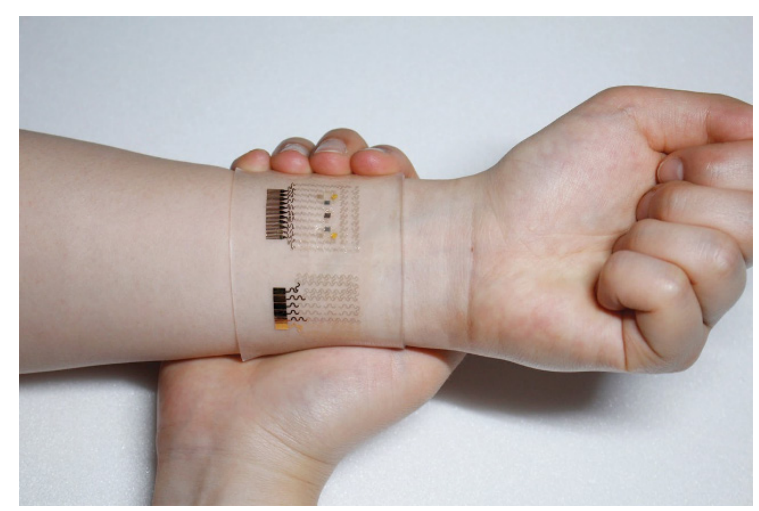

Figure 7. Graphene smart patch for monitoring diabetes [24]

\section{2 mHealth}

In actual aging society, the healthcare system is transformed by mHealth (healthcare using mobile wireless technologies, also called mobile health technologies) that provides new access opportunities to health services and medical care. The advancement in mobile devices (powerful on board computing capability, prebuilt with health sensors, large memories and screens, open operating systems [8] has led to significant growth in the mHealth industry. The latest generation of smartphones makes easier for users to track their health, having a. tremendous potential in assisting seniors and people with chronic conditions to live independently.

Four types of solutions have been identified [9]:

1) Self-healthcare management - focused on the autonomy, implication and self-sufficiency of aging people, without the need for involvement of an external carer in the delivery of the mHealth service. These solutions usually consist of:

- A body sensor network (BSN) - a network of low cost, miniaturized and wireless wearable or implantable interconnected biosensors and actuators that acquire the patient's biosignals and contextual parameters (i.e. ECG, EEG, heart and respiration rates, blood pressure, body temperature, glucose level, spatial location, etc.) [9]. 
- A mobile-based unit (MBU) - in most cases a smartphone that receives the transmitted wirelessly data (through Wi-Fi or shortranged protocols) from sensors and acts as a local storage, processing and analysis unit providing feedback ready for visualization by doctor and patient, through a user-friendly and interactive graphical or audio interface.

An example of an mHealth blood pressure application is presented in Figure 8 .

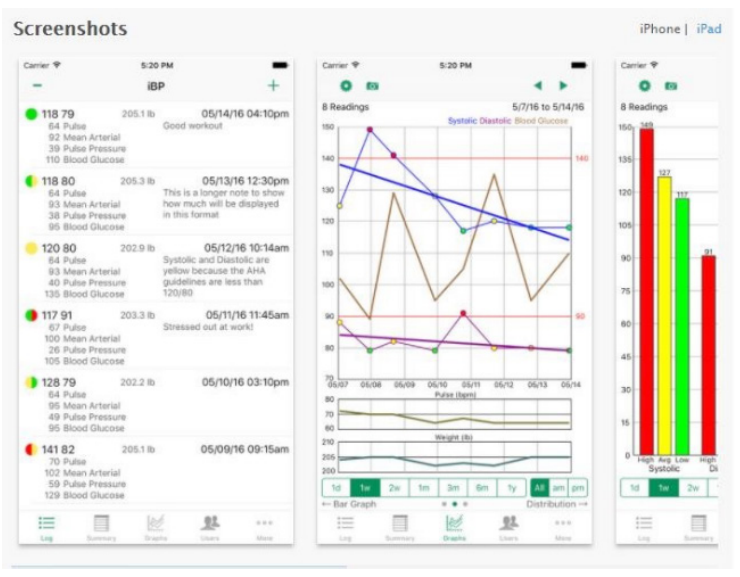

Figure 8. iBP Blood Pressure [15]

2) Assisted healthcare - involves not only the elder in self measurements of health parameters, but an external carer in the process of sharing these data. In case of an emergency, an alarm condition is generated when health parameters exceeded a preset threshold. By using fixed terminals or mobile devices, the carers are warned about undesirable or worrying situations through SMS, voice calls, e-mails, and audio alarms. The data from a certain range are forwarded to the caregiver, including the GPS position of the patient.

3) Supervised healthcare - the data flow between the patient and the caregivers includes all the patient's biosignals, as well as the abnormal data collected in case of an emergency. This data are stored remotely and can be accessed by doctors and families. A monitoring system is also included, involving a remote database where the collected physiological data are periodically sent and stored, allowing doctors, family and friends to manage the patient conditions or to configure the BSN sensors remotely. The carers can remotely access and supervise both current and past physiological data of the senior, evaluate his condition, share and discuss these records with other doctors, set thresholds for sensors and give advice remotely [18].

4) Continuous monitoring - offers a fully automatic analysis of real-time vital biosignals of the patient using a reasoning engine, which proactively uses data mining techniques to correlate data from multiple sensors, assess risk levels and generate preventive actions. Reports are available to both patients and carers. Thus, through the automated healthcare intelligence and a continuous and ubiquitous double sense information flow, not only appropriate actions in response to abnormal situations, but also preventive personalized recommendations are available for supporting both patients and health providers.

Some trends that will reshape the mobile health industry are presented bellow [17]. In 2013, a Research2Guidance report [20] predicted that "2017 would be a significant year for mHealth apps as the market will reach $\$ 26$ billion, with medical apps being integrated into medical professionals' treatment plans".

For syncing all this data into one place it would be necessary:

- Wider IoT connection - The next generation of mHealth technologies will be standalone and will use their own SIM card slots to connect to the internet with high-speed connectivity. Thus, mHealth devices will be able "to connect with other internet-based technologies (smart homes, smart cars, etc.) and create a healthy tech-powered ecosystem for consumers" [17].

- Advanced mHealth technologies - provision of advanced health-assisting features for specific patients, such as in-depth health analysis.

- $\quad$ mHealth business models will be expanded.

Some identified challenges for the mHealth Area are:

- Battery with limited lifetime - Batteries currently cannot withstand a full work day without a recharge [13].

- Multiplatform development - iOS, Windows Phone, and Android being the most common choices. 
- Delays in data transmission and their consequences for patient [21].

- Effort to implement the applications in real environments [12].

\subsection{In-home monitoring systems functioning without disrupting the resident seniors}

For living alone seniors, it can become dangerous as they age when their medical conditions and mobility issues can turn into emergency situations. For making day-to-day life easier and have health monitoring at a click distance without giving up their independence and safety, in our increasingly technology clever society, the most useful products to aid elderly and caregivers are based on innovative technologies.

The remote monitoring systems for the seniors help family members and other caregivers to stay connected to a senior (even from a distance), locate him if he has wandered away or call for help in emergency situations, such as falls.

For improving the monitoring of elderly in their own homes by making it faster and more effective than ever, the healthcare providers and carers are looking for devices increasingly integrated with wireless mobile devices (i.e. wearable sensors that collect real-time health data and are a discreet and comfortable way to collect vital biosignals) and cloud-based data storage that can be accessed by physicians at any time. Medical alert bracelets and systems communicate with a monitoring station, so if a senior is in trouble, he can call for help. They combine both sensor technology and personal emergency response to help promote senior's independent life. See Figure 9.

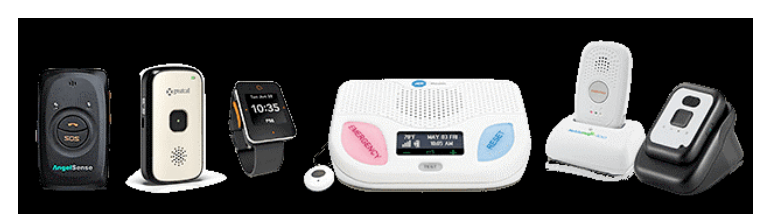

Figure 9. Examples of medical monitoring and alert systems [16]

The devices are designed to identify problems before they become emergencies by observing daily activity patterns and alerting caregivers if any abnormalities are noticed. Alerts to caregivers are provided by the devices without disrupting residents and can be either phone calls, text messages, and/or emails letting the caregiver know when events happen.

In the age of advancing technologies, seniors can be monitored remotely by family and caregivers from any computer, tablet or smartphone. See Figure 10.

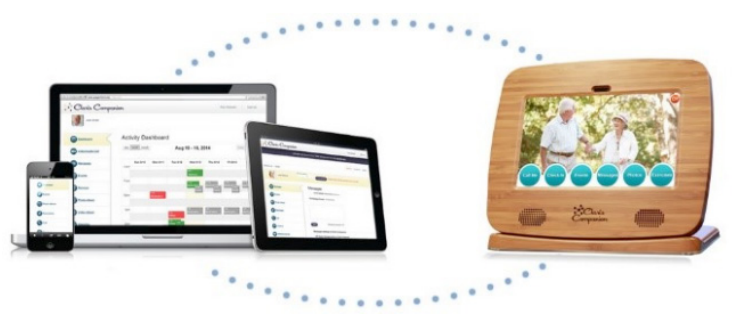

Figure 10. An example interface of a senior's remote monitoring [14]

The 24/7 remote monitoring facilities are combined in one simple unit integrating the best features of a tablet, computer, digital picture frame, mobile phone. Touch-screen monitor systems with easy to use interface for the seniors that offer a simple way to video chat, message, send photos, view scheduled appointments are well appreciated by seniors.

A combination of in-home monitoring technology, simple medical devices and a helpful human element will provide the balance between service usefulness and protection of senior's dignity. Smart homes could reduce the challenges associated with aged care while affording safety and wellbeing for seniors and families.

\section{Case study: MSI-MDD project}

The "Conceptual Model for a Platform of Integrated Services based on Cloud for Home Monitoring of Seniors affected by Dementia (MSI-MDD)" project proposed a conceptual model of an integrated platform for cloudbased services that were designed to create a safe familiar environment and an appropriate personalised health management for the seniors suffering from dementia. Additional services for sustaining improvements of specific healthcare provided by physicians or carers were integrated with the senior-centred ones that enable a real time monitoring of the patient, with a 
strong emphasis on crisis prevention and delay progressive deterioration of health.

MSI-MDD platform is structured into five main components presented in the application layer (see Figure 11).

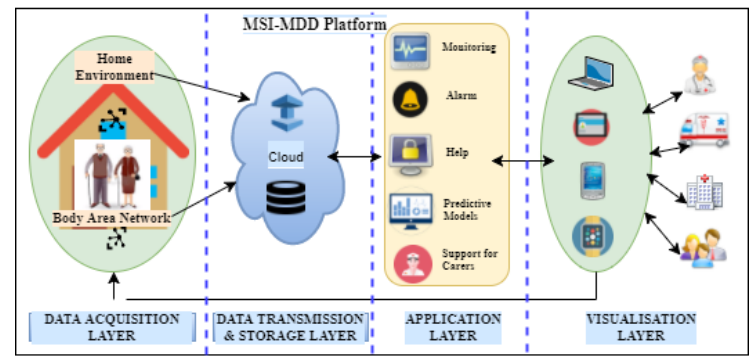

Figure 11. Current architecture of the MSI-MDD Platform

- Help: it provides support to daily activities such as locating a lost object - by activating an online function that triggers a sensor beep. Requesting help or the presence of acarer/ family member in case of an unexpected event is another example of comprised service.

- Monitoring: it enables the collecting of data and information regarding the senior's health condition by using medical devices and sensors. Data is stored in a patientcentred database and it can be used both for the personal health management, and for developing predictive models. The identified dysfunctions and the abnormal values of some measured parameters initiate a procedure whereby the carer and the attending physician are warned in real time.

- Alarm: senior's carers are warned both locally and remotely in case of a malfunction of appliances or of parts of the space environment.

- Predictive Models: they are developed based on the data and information provided by the Monitoring component. Predictive models aim to allow a preventive and proactive health management for a personalized senior care.

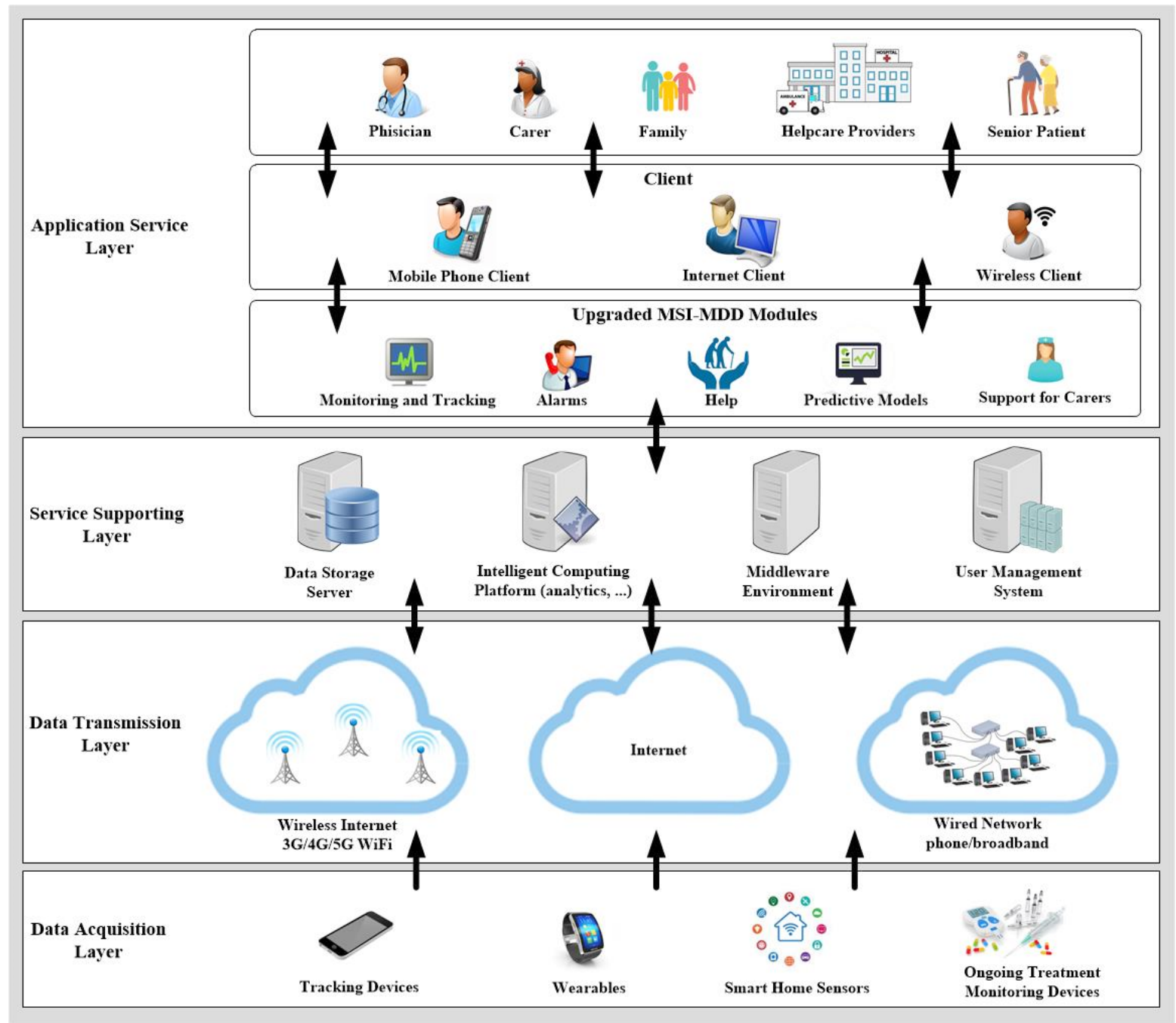

Figure 12. New proposed architecture 
- Support for Carers: it contains modules with updated and practical information for carers to improve and better understand their duties in taking care of seniors affected by dementia. In order to enhance the efficiency of the training process, the used eLearning system has to be adaptive and personalised on the specific profile of the students, namely senior's carers [2], [4].

\section{A proposed upgraded architecture for MSI-MDD platform}

The solution provided by MSI-MDD project and designed for the seniors affected by dementia can be extended and adapted to different categories of elderly persons.

In this respect and taking into consideration the opportunities brought by the new assisted technology, the MSI-MDD platform can be further upgraded with new functionalities. The proposed new architecture is shown in Figure 12. Some of the envisioned functions are presented as it follows:

Firstly, taking into consideration the associated comorbidities, the vital signs monitoring that is based on the newest wearable technologies (like smart contact lens or graphene smart patch), together with the use of predictive models (allowing $a$ preventive and proactive disease management for a personalized care of the patient) can be of interest for all categories of seniors (as illustrated in Data Acquisition Layer in Figure 12).

Secondly, the access by mobile phones to healthcare data and to the management of age associated diseases is valuable not only for seniors, but also for a coordinated healthcare delivery among every person directly involved in the life of the elderly. The Data Acquisition and Application Service Layers from Figure 12 illustrate how a mHealth solution can add up the previous assisted healthcare services of MSI-MDD.

Thirdly, MSI-MDD platform can be extended with another functionality regarding the localization of wandering senior patients inside hospitals or nursing homes by using radio-frequency identification (RFID) sensors or outside the house by using GPS facilities for increasing elderly's safety and assuring the possibility of his/her free movement. (as illustrated in Application Service Layer in Figure 12).

In conclusion, MSI-MDD platform can be further deployed in order to become an efficient support to other categories of seniors and to provide improved remote senior-centred healthcare services. A progressively enlarged range of assistive technologies integrated in the familiar environment can better address the specific needs of the elderly.

\section{Acknowledgements}

"Conceptual Model for a Platform of Integrated Services based on Cloud for Home Monitoring of Seniors affected by Dementia" (MSI-MDD) project was funded by Ministry of Research and innovation (2014-2015). The authors gratefully acknowledge the contribution of the Romanian ministry.

\section{REFERENCES}

1. AALIANCE2 European Next Generation Ambient Assisted Living Innovation Alliance (2014). Ambient Assistive Living Roadmap 2014, Available at http://cordis.europa.eu/docs/projects/ cnect/5/288705/080/deliverables/001 AA2WP2D 2 7aRM2rev41.pdf.

2. Alexandru, A., Tîrziu, E., Tudora, E. \& Bica, O. (2015). Enhanced Education by Using Intelligent Agents in Multi-Agent Adaptive e-Learning Systems, Studies in Informatics and Control, ISSN 1220-1766, 24 (1), 13-22.
3. Alford, K. \& Johnston, R., (2011). Report of the Industry Uptake of Enabling Technologies Foresight Workshop: Enabling Assistive Technologies for Aged Care, 3-16.

4. Băjenaru, L., Smeureanu, I. \& Balog, A. (2016). An Ontology-Based E-Learning Framework for Healthcare Human Resource Management, Studies in Informatics and Control, ISSN 1220-1766, 25(1), 99-108.

5. Berger, M., (2017). Smart graphene contact lenses bring wearable electronics to the eye, 
Nanowerk, Available at http://www.nano werk.com/spotlight/spotid=46780.php.

6. Blackman, S., Matlo, C., Bobrovitskiy, C., et al. (2015). Ambient Assisted Living Technologies for Aging Well: A Scoping Review. Journal of Intelligent Systems, 25(1), 55-69.

7. Bonato, P. (2010). Wearable sensors and systems. From enabling technology to clinical applications. IEEE Eng Med Biol Mag, 29.

8. Boulos, M., Wheeler, S., Tavares, C. \& Jones, R. (2011). How smartphones are changing the face of mobile and participatory healthcare: An overview, with example from eCAALYX, BioMedical Eng. OnLine, 10(1), 24

9. Chiarini, G., Ray, P., Akter, S., Masella, C. \& Ganz, A. (2013). mHealth technologies for chronic diseases and elders: a systematic review, IEEE Journal on Selected Areas in Communications, 31(9), 6-18.

10. Ericsson ConsumerLab (2017) Report: From Healthcare to Homecare, Available at https://www.ericsson.com/en/networkedsociety/trends-and-insights/consumerlab/co nsumer-insights/reports/transforming-heal thcare-homecare.

11. European Commission, Directorate-General for Communications Networks, Content and Technology (2016). Smart Wearables: Reflection and Orientation Paper, 5.

12. Gomes, A.T.A., Ziviani, A., Correa, B.S.P.M., Teixeira, I.M. \& Moreira, V.M. (2012). SPLiCE: a software product line for healthcare, In Proceedings of the 2nd ACM SIGHIT International Health Informatics Symposium, ACM, 721-726.

13. Heslop, L., Weeding, S., Dawson, L., Fisher, J. \& Howard, A. (2010). Implementation issues for mobile-wireless infrastructure and mobile health care computing devices for a hospital ward setting, Journal of medical systems, 34(4), 509-518.

14. http://www.assistedlivingtechnologies.com/2remote-monitoring-elderly

15. https://itunes.apple.com/gb/app/ibp-bloodpressure $/ \mathrm{id} 306526794$ ? $\mathrm{mt}=8$.

16. https://www.safewise.com/resources/medicalalert-devices
17. JenTechnology (2017). Important Trends That Will Reshape mHealth in 2017, Available at http://conscienhealth.org/ 2017/02/importanttrends-will-reshape-mhealth-2017/.

18. Lv, Z., Xia, F., Wu, G., Yao, L. \& Chen, Z. (2010). iCare: a mobile health monitoring system for the elderly, In Proceedings of the 2010 IEEE/ACM Int'l Conference on Green Computing and Communications \& Int'l Conference on Cyber, Physical and Social Computing, IEEE Computer Society, 699-705).

19. Mokaba, C. (2016). Thread-based diagnostic platform could be effective substrate for latest diagnostic devices and smart wearable systems, research suggests, Available at http:// newstonight.co.za/ content/thread-baseddiagnostic-platform-could-be-effectivesubstrate-latest-diagnostic-devices-and-

20. Researche2Guidance (2017). The market for mHealth app services will reach $\$ 26$ billion by 2017, Available to https://research2guidance. com/the-market-for-mhealth-app-serviceswill-reach-26-billion-by-2017/.

21. Soomro, A. \& Schmitt, R. (2011). A framework for mobile healthcare applications over heterogeneous networks. In e-health networking applications and services (Healthcom), 2011 13th IEEE international conference on, 70-73.

22. United Nations, Department of Economic and Social Affairs, Population Division (2017) World Population Prospects: The 2017 Revision, Key Findings and Advance Tables. Working Paper No. ESA/P/WP/248, 19.

23. Wang, Z., Yang, Z., \& Dong, T. (2017). A review of wearable technologies for elderly care that can accurately track indoor position, recognize physical activities and monitor vital signs in real time. Sensors, 2.

24. Wong, S. (2016). Graphene smart patch for monitoring diabetes could save lives, Newscientist, Available at: https://www. newscientist.com/article/mg22930661-900smart-patch-for-diabetes/.

25. Yoon, S., Sim, J., Cho, Y. (2016). A Flexible and Wearable Human Stress Monitoring Patch Scientific Reports 6, Available at https://www. nature.com/ articles/ srep23468. 